\title{
Not Knowing What One Knows: A Meaningful Failure of Metacognition in Capuchin Monkeys
}

\author{
Travis R. Smith ${ }^{1}$, J. David Smith ${ }^{1,2}$, and Michael J. Beran ${ }^{1,2}$ \\ ${ }^{1}$ Language Research Center, Georgia State University, Atlanta, GA \\ ${ }^{2}$ Psychology Department, Georgia State University, Atlanta, GA \\ *Corresponding author (Email: smith.travis.r@gmail.com)
}

Citation - Smith, T. R., Smith, J. D., \& Beran, M. J. (2018). Not knowing what one knows: A Meaningful failure of metacognition in capuchin monkeys. Animal Behavior and Cognition, 5(1), 55-67. https://doi.org/10.26451/abc.05.01.05.2018

\begin{abstract}
Metacognition encompasses the processes of monitoring representational and perceptual states and controlling information-gathering behaviors. Metacognition is considered one of humans' most sophisticated abilities, and it has been a growing area of focus in comparative cognition research. Despite the successes of some species such as the great apes and some Old World monkeys, there has been a fairly consistent lack of metacognitive responding in the New World primate species, capuchin monkeys. These failures are meaningful for what they highlight about the phylogenetic breadth of metacognition, and for what they offer to ongoing debates about the proper interpretation of data from other species that do succeed in various tests of comparative metacognition. We summarize these meaningful failures and place them in a broader context of comparative metacognition research, with a specific focus on explaining what it might mean that some monkeys seemingly do not know what they know.
\end{abstract}

Keywords - Metacognition, Uncertainty monitoring, Information seeking, Capuchin monkeys

Metacognition is the monitoring of one's own cognitive states, such as when someone is aware of whether they know (or remember) the appropriate response in a situation. This awareness is considered a private form of introspection that is an element of what we understand as consciousness. Metacognition is well-studied in adult humans (Dunlosky \& Bjork, 2008; Flavell, 1979; Metcalfe \& Kober, 2005; Nelson, 1996; Nelson \& Narens, 1990; Schwartz, 2008). Research on the metacognitive capacity of nonhuman animals (hereafter, animals) is motivated, in part, by the hope to discover the rudimentary cognitive processes underlying metacognitive capacities in humans. The discovery of metacognitive processes in animals could help clarify the basic mechanisms underlying these processes; for instance, the degree to which metacognitive abilities can function without the mediation of language and culture. Furthermore, research in animal metacognition may help us develop a better understanding of the private world of animals. By one view, metacognition does not, perhaps cannot, exist outside of humans (Carruthers, 2008). By another view, animals might experience the world, even the introspective-reflective world, much as humans do (see Beran, Brandl, Perner, \& Proust, 2012; Maestripieri, 2003; Vonk \& Shackelford, 2012; Zentall \& Wasserman, 2012). Research into animal metacognition furthers this debate. Finally, this research allows us to trace metacognitive psychological capacities across species to help refine theories regarding the evolutionary emergence of this capacity.

Animal research in metacognition has generated behavioral data that support the claim that some nonhuman primates, such as macaques (Macaca mulatta) and chimpanzees (Pan troglodytes), possess a 
metacognitive awareness of their own knowledge states (for reviews, see Smith, Beran, Couchman, Coutinho, \& Boomer, 2009a; Smith, Couchman, \& Beran, 2012, 2014); however, for the past decade the evidence of metacognitive abilities in capuchin monkeys (Sapajus apella) has been mixed (Smith, Beran, Couchman, Coutinho, \& Boomer, 2009b). We propose here that this is a meaningful "failure" that has broad implications for the issues raised above and for comparative methodologies in studying metacognition. This paper will review the state of research in capuchin metacognitive abilities, discuss the consistent theme of mixed or null results in the literature, and consider possible implications for understanding animal and human metacognition.

\section{Successes in Animal Metacognition Tests - Primarily Primates}

The early experiments designed to test for animal metacognition started with a dolphin (Smith et al., 1995) and rhesus macaques (Shields, Smith, \& Washburn, 1997; Smith, Shields, Schull, \& Washburn, 1997). For example, Smith et al. (1997) trained monkeys on a primary sparse/dense pixel discrimination task using a computer interface system that allowed the monkeys to use a joystick to move a cursor and make selections in classifying a stimulus as being dense or sparse in pixel density (Figure 1A). The pixel density of the sample varied across trials with intermediate pixel density samples that were difficult to categorize psychophysically. A secondary "uncertain response" (UR) was made available for monkeys to decline any trial and proceed to an easier trial without the food reward that followed a correct response or a long timeout that followed an incorrect response. The monkeys' use of the UR increased on the intermediate pixel density trials and peaked near where sparse and dense categorizations occurred with equal likelihood, and the risk of making one of those classification errors was greatest (Figure 1C \& 1D). Humans also were tested using the same procedure and produced nearly identical results (Figure 1B), and they also self-reported that they were consciously uncertain of the appropriate categorization for those trials, suggesting that this paradigm captures what people mean when they state they "feel uncertain." These data begin to support a claim that monkeys experience a private sense of uncertainty and this allows them to avoid the problems for which they do not have solutions.

However, the weight of such a claim could not be supported by those data alone. Those URs might not be a product of a private event, such as metacognitive awareness of uncertainty, that operates independently of (but in conjunction with) public stimulus-response learning processes (Hampton, 2009). For example, they could be reactions to environmental cues (e.g., monkeys' memorizing the stimuli that occasion negative consequences and subsequently using the UR to avoid those consequences), behavioral cues (e.g., monkeys' latencies from hesitation are used as the discriminative stimulus for the uncertain response), and response competition (e.g., monkeys' responding to the uncertain option because the alternative options are equally unattractive). Given these possible alternative mechanisms, URs in animals would not necessarily reveal the presence of an introspective awareness of their knowledge state.

To gather more evidence supporting an account for animal private metacognition, researchers have conducted experiments with controls that minimize the likelihood that alternative explanations may account for the data. Researchers have also arranged a variety of different procedures that make predictions about animal performances assuming they possess metacognitive abilities. For example, in Smith et al. (1997), it is possible that the UR was used as a proxy categorization response for intermediate densities. To address this, Shields et al. (1997) evaluated whether monkeys and humans would use the UR when engaging in a same/different relative discrimination task, where macaques did not classify a specific density level, but chose whether two pixel-density magnitudes were equivalent. This procedure did not allow the monkey (or human) to treat the UR as a categorization response tied to a given class of stimuli (e.g., like an intermediate pixel density). Yet again, humans (Figure 2A) and monkeys (Figure 2B) displayed an increase in the use of the UR corresponding with a decrease in use of the categorization responses for the most difficult trials. Other experiments explored metacognition in rhesus monkeys and showed that the use of the UR or other metacognitive responses transfers across a variety of different tasks (Basile, Schroeder, Brown, Templer, \& Hampton, 2015; Brown, Templer, \& Hampton, 2017; Kornell, Son, \& Terrace, 2007; Smith, Redford, Beran, \& Washburn, 2010; Templer \& Hampton, 2012) 
and that monkeys will continue using the UR when feedback is delayed (Smith, Beran, Redford, \& Washburn, 2006), minimizing the likelihood that task-specific environmental cues are responsible for the use of the UR.

\section{A. Trial Screen}
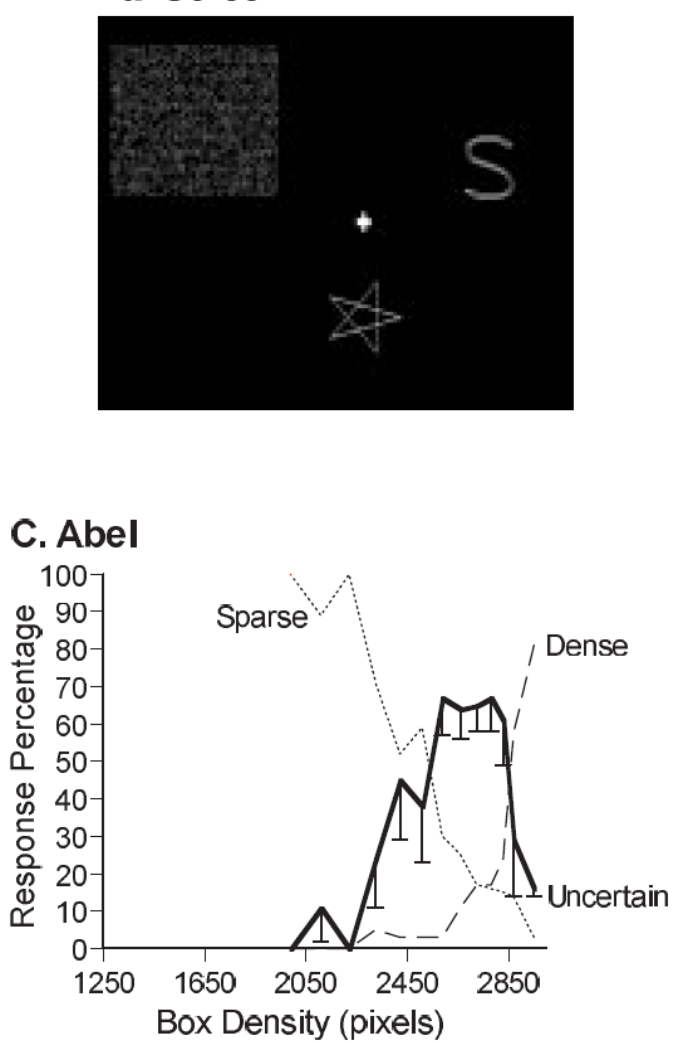

\section{B. Humans}

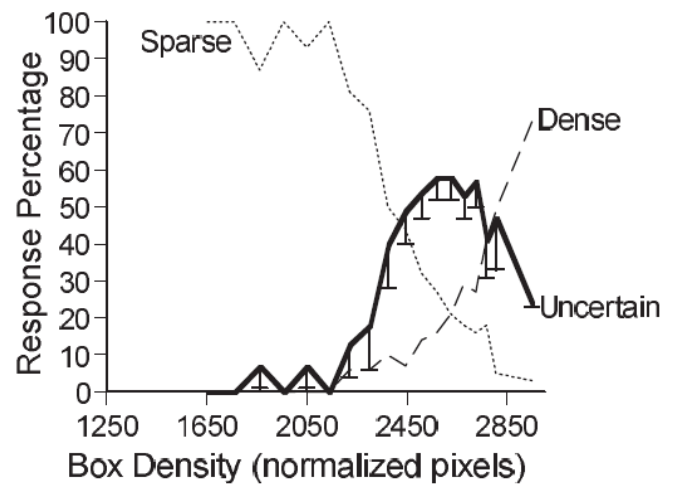

D. Baker

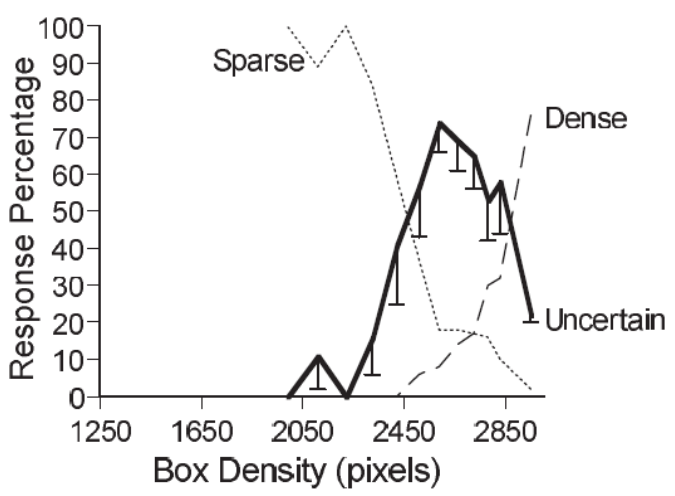

Figure 1. A. An example trial in the dense-sparse discrimination task given to humans and to monkeys. B. The performance of seven humans in the dense-sparse task. The dense response was correct for boxes with exactly 2,950 pixels - these trials are shown at the far right. All other boxes required the sparse response. The horizontal axis indicates the normalized pixel-density of the box. The solid line represents the percentage of trials receiving the Uncertain response at each density level. The percentages of trials ending with the dense response (dashed line) or sparse response (dotted line) are also shown. C. The performance of one monkey named Abel in this task. D. The performance of another monkey, Baker, in this task. From "The Comparative Psychology of Uncertainty Monitoring and Metacognition,” by J. D. Smith, W. E. Shields, and D. A. Washburn, 2003, Behavioral and Brain Sciences, 26, p. 322. Copyright 2003 by the Cambridge University Press. Reprinted with permission.

If monkeys genuinely demonstrate metacognition through their behavior, then such metacognition should express itself across a variety of different tasks that provide converging evidence to support the weight of the claim for private metacognitive capacities in animals. Such tasks include the uncertainty response already discussed (e.g., Smith et al., 1997), information-seeking responses in computerized tasks (e.g., Beran \& Smith, 2011), responses that show confidence in prior decisions (e.g., Beran et al., 2015), and metamemory tasks in which the animal may have forgotten some previously learned material, and may need to decline a memory test or to seek out additional information (e.g., Basile et al., 2015; Hampton, 2001; Marsh \& MacDonald, 2012a). 

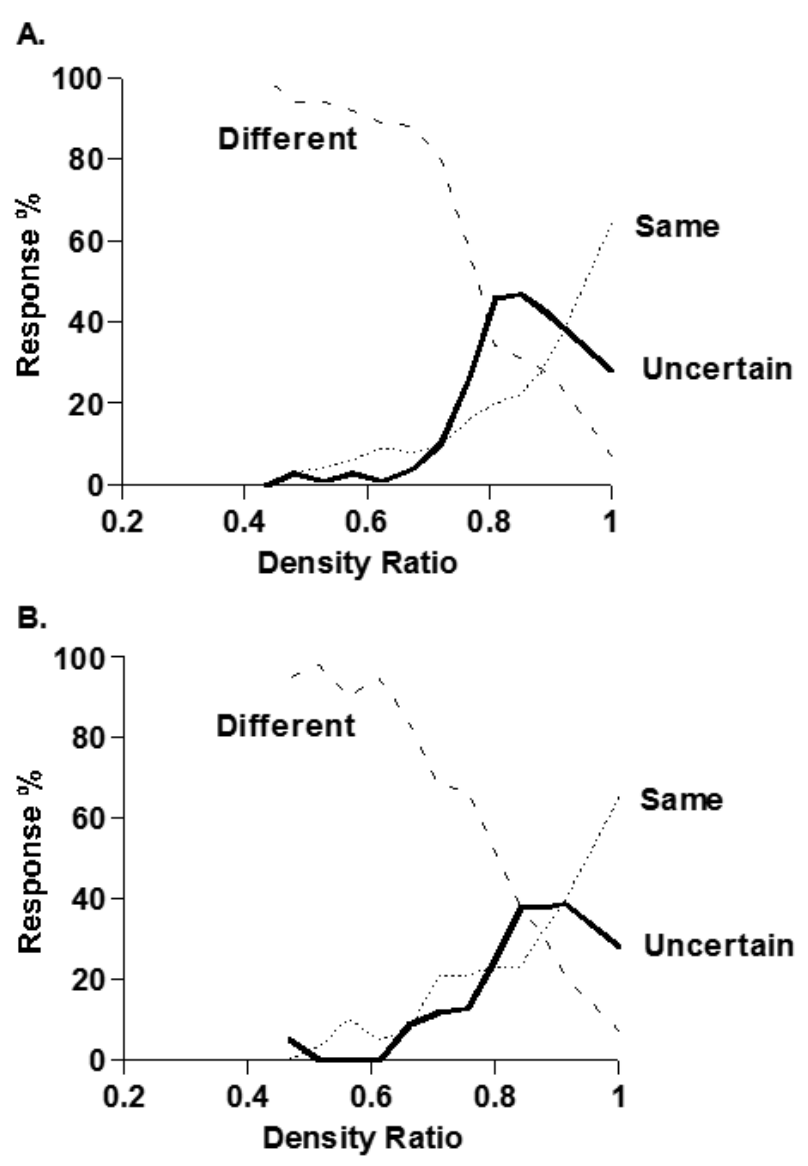

Figure 2. Composite performance by (A) six humans and (B) two monkeys in a same-different task. The horizontal axis gives the ratio between the densities of the comparison box and the standard box for trials of different disparities. The same response was correct for trials at a proportional box disparity of 1.0. These trials are represented by the rightmost data points. All other trials required the different response. The solid line represents the percentage of trials receiving the uncertainty response at each density ratio. The percentages of trials ending with the different response (dashed line) or same response (dotted line) are also shown. From "Uncertain Responses by Humans and Rhesus Monkeys (Macaca mulatta) in a Psychophysical Same-Different Task," by W. E. Shields, J. D. Smith, and D. A. Washburn, 1997, Journal of Experimental Psychology: General, 126, p. 158. Copyright 1997 by the American Psychological Association. Reprinted with permission.

Information-seeking tasks involve introducing subjects to trials in which they sometimes need to proactively seek visual information to observe in order to correctly respond. For example, Call (2010) and Call and Carpenter (2001) demonstrated that chimpanzees and children correctly responded to a memory game based on what they knew and did not know. When shown where food was hidden in an array, they immediately pointed toward it, but when not shown they took the time first to look before making a choice. Subsequent work with orangutans highlighted that such differential responses were not made on the basis of simple learned contingencies but rather seemed to reflect what was at stake, and what could be known by the animals at the initial time when a choice could be made or more information could be obtained (Marsh \& MacDonald, 2012a, b). Other apes also showed good evidence of memory monitoring, and adaptive use of trial-decline responses on difficult memory tests (e.g., Suda-King, 2008; Suda-King, Bania, Stromberg, \& Subiaul, 2013). Beran, Smith, and Perdue (2013) designed a task in which chimpanzees had to report what was in a box using a lexigram communication system. In some conditions the chimpanzees saw the object, and in other conditions they did not and would have to travel to inspect the contents of the box prior to making a report. The chimpanzees immediately reported the contents of the box when they were shown those contents but traveled to inspect the contents of the box when they 
were not shown its contents, or when they were shown a food item, but from a different box. These results were consistent with a metacognitive interpretation that the chimpanzees' inspection was due to awareness that they lacked the necessary information to adequately respond.

If an individual is metacognitively aware, then that individual typically can flexibly tailor the amount of uncertainty risk they are willing to assume, based on the amounts of gains and losses before them. In Zakrzewski, Perdue, Beran, Church, and Smith (2014), macaques engaged in a primary pixeldensity computerized discrimination task to accumulate token rewards that could be "cashed in" for food pellets. If the monkeys made a correct response they would accumulate more tokens and if they made an incorrect response they would lose all accumulated tokens. The macaques were more likely to "cash in" tokens when they were facing a challenging forthcoming discrimination trial (suggesting that they were metacognitively aware of the consequences for accepting that trial), and they were more likely to reject challenging trials after they had already accumulated many tokens (suggesting that they were also sensitive to the level of risk involved in accepting a challenging trial; Figure 3). These results nicely matched those from other prospective judgments made by macaques. In these judgment tasks, monkeys also had to accumulate digital rewards before receiving real rewards, and monkeys showed that they learned when to make a primary response and when to avoid that response. These patterns transferred to multiple tasks (Kornell et al., 2007). In fact, macaques showed both retrospective and prospective metacognitive judgments using this approach (Morgan, Kornell, Kornblum, \& Terrace, 2014). Brown et al. (2017) thoroughly investigated the capacity for macaques to generalize the use of an UR, declining the test and accepting fewer pellets, across a variety of tasks. This includes generalizing the use of the UR across a size discrimination task, a brightness discrimination task, an arch-length discrimination task, a line orientation discrimination task, a metamemory task, and a prospective metamemory task. The monkeys successfully transferred the use of the UR across the tasks, this transfer was observed in the first test session, and the UR-transfer was more successful across successive task exposures (also see Basile et al., 2015).

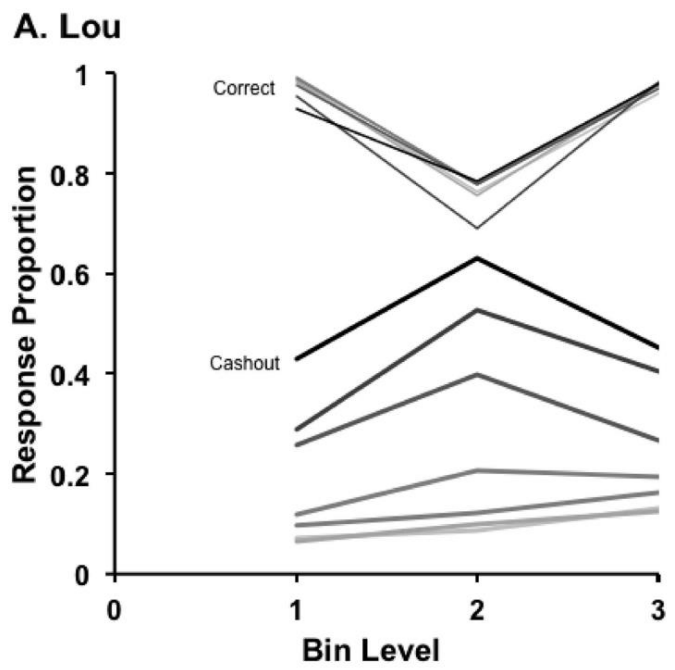

\section{B. Murph}

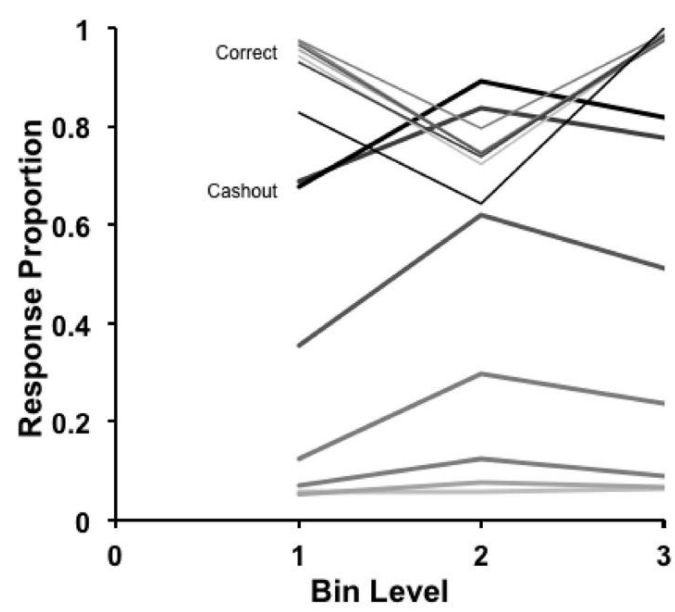

Figure 3. Results from monkeys (A) Lou and (B) Murph in a task with variable numbers of rewards at risk and the need to make a density discrimination. Bin 1 includes the sparsest trials; Bin 2 includes trials that are intermediate between sparse and dense; Bin 3 includes the densest trials. The peaked curves show the proportion of all trials at each bin level receiving the cashout response, which allowed monkeys to collect accumulated rewards before they had to make the density discrimination. The troughed curves show the proportion of correct responses (for all trials completed directly without cashing out). For both cashout responses and proportion correct, progressively darker lines depict increasingly risky situations in which there were more coins/rewards at stake. Reprinted from "Cashing Out: The Decisional Flexibility of Uncertainty Responses in Rhesus Macaques (Macaca mulatta) and Humans (Homo sapiens)," by A. C. Zakrzewski, B. M. Perdue, M. J. Beran, B. A. Church, and J. D. Smith, 2014, Journal of Experimental Psychology: Animal Learning \& Cognition, 40, p. 494. Copyright 2014 by the American Psychological Association. Reprinted with permission. 
Other metamemory tasks, such as that developed by Hampton (2001), also provide a strong test against environmental cues controlling the UR. Hampton had macaques work in a delayed matching-tosample task and reported that the monkeys avoided taking more tests at long delay intervals when the sample was most likely to have been forgotten, but also on trials in which the sample was never presented. Thus, the macaques reported uncertainty in their recall under conditions in which no single environmental stimulus could easily cue the UR response and when the likelihood of correctly responding on the task was small. Templer and Hampton (2012) also presented rhesus monkeys with a set of memory tasks and found that the monkeys were consistently more accurate on trials where they could have avoided answering the primary question than on trials where they were forced to answer it. And, the monkeys adjusted their use of trial declining responses depending on whether the various tasks made it easier or harder to remember stimuli.

\section{Limited Success in Metacognition Tests in Non-Primate Species}

Despite the substantial evidence for potential metacognition reported in apes, dolphins, and macaques, not all species have clearly demonstrated metacognitive responding that cannot be attributed to other causes. Rats (Foote \& Crystal, 2007, 2012) and birds (Goto \& Watanabe, 2012; Inman \& Shettleworth, 1999; Roberts et al. 2009; Teller, 1989) have provided inconclusive evidence supporting a claim of metacognitive abilities in some experiments. In other experiments, rats (Kirk, McMillan, \& Roberts, 2014; Templer, Lee, \& Preston, 2017) and birds (Castro \& Wasserman, 2013; Roberts, McMillan, Musolino, \& Cole, 2012; Watanabe \& Clayton, 2016) do appear to show responding consistent with a hypothesis of influence by a metacognitive system using the information-seeking paradigm or an uncertainty response. However, alternative associative hypotheses have not yet been fully ruled out (Zentall \& Stagner, 2010). And while it might be tempting to assume that metacognitive abilities will be consistently found in the primate lineage, the lack of good consistent evidence from capuchin monkeys complicates this view (Smith et al., 2009b).

\section{The Curious Case of Capuchin Monkeys as a Meaningful Failure of Metacognition}

Capuchin monkeys have been tested in a variety of tasks to determine whether they display the same potentially metacognitive behaviors that other nonhuman primates have demonstrated. Paukner, Anderson, and Fujita (2006) presented capuchin monkeys with tubes that may have contained food and reported that their visual searching of the tubes was not "rational" in the sense that they needlessly searched transparent tubes and attempted to search bent tubes that would never provide the necessary information. Thus, those monkeys' searching behavior was not consistent with the claim that they were aware of whether they knew the tube was baited or not; however, the capuchin monkeys may have also manipulated the tubes for reasons independent of information seeking. Basile, Hampton, Suomi, and Murray (2009) tested capuchin monkeys in a paradigm that had successfully demonstrated metacognitivelike behavior in apes (Call \& Carpenter, 2001; Call, 2010) and rhesus macaques (Hampton, Zivin, \& Murray, 2004). In this task, food was concealed in one of a series of tubes and in some trials the food baiting was observable and in other trials it was concealed. Apes, macaques, and 2.5-year-old human children adaptively examined the tubes when they did not see the baiting or when there was a delay between baiting and retrieval (i.e., they may have forgotten), and they did not examine the tubes when they had fresh information about the food location (Call \& Carpenter, 2001). Capuchin monkeys, however, failed to respond adaptively in this experiment (Basile et al., 2009). Initially they did not examine the tubes when they should have. Following remedial training they examined the concealed tubes more than the baited tubes, but they still tended to examine the baited tubes over half the time. Finally, once there was an added effort cost to examining the tubes the monkeys searched the tube less regardless of whether they observed the baiting. Thus, there was limited evidence in favor of the monkeys showing metacognition and control processes. 
Vining and Marsh (2015) arranged a capuchin information-seeking experiment in which they found that capuchin monkeys met some but not all of the predictions that support a claim of metacognitive monitoring. The monkeys had to locate food hidden under a cup and had the opportunity to examine the cups by looking beneath the transparent Plexiglas tray holding the cups. Generally, the monkeys had a bias to crouch and examine the contents of the cup prior to making a selection across conditions. However, two out of three capuchin monkeys were less likely to examine the cups when they saw the food baiting or when food was located beneath a transparent cup. Despite this, the monkeys also failed to use inference by exclusion (i.e., if the food was not under the transparent cup it was always under the alternative occluded cup) to select the appropriate cup without first examining both cups. Thus, while monkeys showed a greater tendency to investigate cups when they lacked information, they still also tended to investigate the cups unnecessarily. Vining and Marsh suggested that capuchin monkeys may have a basic metacognitive capacity when dealing with perceptual information (i.e., seeing or have seen food baiting), but at least a less developed capacity for cognitive information (i.e., abstract uncertainty stemming from not knowing a discrimination response). Marsh (2014) reported that lion-tailed macaques (Macaca silenus) also searched cups possibly containing food when inference by exclusion would have been informative enough to make that response unnecessary. Marsh argued that the search task may not solely assess metacognition, but also possibly assesses a generalized search response. This may also partly explain unnecessary searching in capuchin monkeys.

Beran and Smith (2011) assessed information seeking using a computerized task with capuchin monkeys and rhesus macaques and contrasted performances with published pigeon results (Roberts et al., 2009). The primary task was a matching-to-sample task with three comparison options. Occasionally the sample stimulus and/or comparison stimuli were occluded and the secondary information-seeking task included two options - one that revealed the sample stimulus and another that revealed the comparison stimuli. Four different trial types included: (1) both sample and comparison stimuli were present and the appropriate response was to ignore the stimulus-revealing options and to correctly match; (2) neither sample nor comparison stimuli were present and the appropriate response was to first reveal the sample, then reveal the comparison stimuli to match, and finally to correctly match; (3) the comparison stimuli, but not the sample stimulus, were present and the appropriate response was to reveal the sample (and not the comparison stimuli) prior to attempting to match; (4) the sample stimulus, but not the comparison stimuli, were present and the appropriate response was to reveal the comparison stimuli (and not the sample) prior to attempting to match. Capuchin monkeys and macaques, in contrast to pigeons, learned to reveal the sample and then reveal the comparisons prior to matching. However, while four out of eight macaques made all of the appropriate responses for the above trial types, none of the capuchin monkeys made appropriate responses for all of those trial types. Three of the seven capuchin monkeys did pass three of the trial types (types 1, 2, 4). Thus, capuchin monkeys provided some evidence supporting metacognition, but, consistent with prior research, they failed to pass all of the arranged tests.

Capuchin monkeys have also had their metacognitive abilities assessed using the common trialdecline method. In a metamemory study, Fujita (2009) arranged a delayed matching-to-sample task. Following the variable forgetting interval $(2,4,8,16 \mathrm{~s})$, but prior to the comparison, the monkeys had the opportunity to escape the trial with a response that would deliver the reward food at a reduced probability. Capuchin monkeys showed a systematic increase in the frequency of using the escape response on longer delay trials for which accuracy was lower. Furthermore, they were more likely to escape the trial on special trials in which the sample was never presented (and therefore could not be remembered). These results are similar to Hampton's (2001) study with macaques and support the claim that the capuchin monkeys were aware of their own ignorance about the correct response. But there were still limitations that complicated that interpretation. First, while capuchin monkeys were more likely to escape difficult trials, they still frequently $(\sim 40-50 \%)$ accepted trials in which they were likely to produce an incorrect response. Second, only one of two capuchin monkeys showed higher accuracy on the trials they chose to complete compared to the trials that they were forced to complete. If the capuchin monkeys were correctly monitoring a valid psychological signal like the confidence of their memory knowledge, then they should have performed better on the trials they opted to complete. However, it is worth noting that 
one macaque (out of two) in Hampton's study also escaped $60 \%$ of the time when no sample was shown, and that same macaque also did not perform better on freely chosen trials. Thus, it would be unfair to make a strong claim that all macaques clearly show a metacognitive-like effect here while capuchin monkeys do not.

In an experiment similar to Smith et al. (1997) with macaques, Beran, Smith, Coutinho, Couchman, and Boomer (2009) arranged a sparse/middle/dense (SMD) pixel discrimination task interleaved with a sparse/uncertain/dense (SUD) task. In the SMD task, capuchin monkeys could receive food rewards for correctly categorizing pixel boxes as sparse, intermediate, or dense in lit pixels. In the SUD task, rewards were given only for correctly categorized sparse or dense pixel boxes, but capuchin monkeys could decline any trials using the UR. Overall, the capuchin monkeys effectively used the "Middle" response to categorize intermediate pixel densities, but they did not use the UR, even when the consequence for an incorrect response was a considerable time-out that cost them many opportunities to earn pellets (Figure 4). This demonstrated that capuchin monkeys do not use the uncertain response to decline difficult trials in the same way that macaques do. However, it was interesting that the capuchin monkeys also did not use the UR even though the negative consequence for an incorrect response was so high. This suggests that while capuchin monkeys did not use the UR to express uncertainty, associative mechanisms also did not encourage the use of the UR for the utility of reward rate maximizing. This dissociation between using the UR as a categorization response, but not as a trial escape response in capuchin monkeys, further suggests that the metacognitive performances observed in macaques using the UR escape response is not easily explained the monkeys treating the UR simply as another categorization response. Otherwise, the capuchin monkeys should have been successful using the escape response.
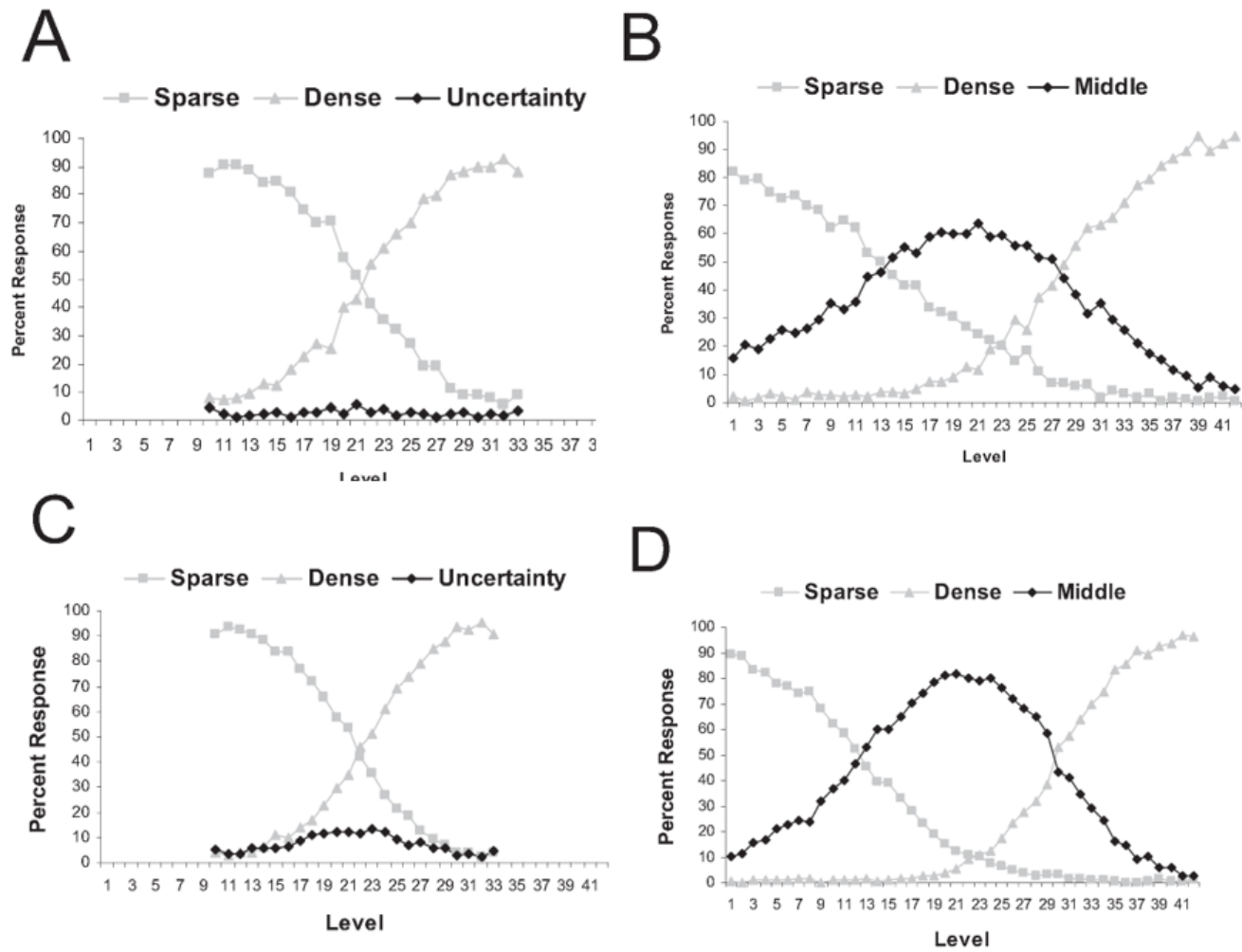

Figure 4. Performance in Experiment 2 of Beran et al. (2009) by task order completion. The top row shows performance for monkeys who completed the SUD task (A) followed by the SMD task (B). The bottom row shows performance for monkeys who completed the SMD task (C) followed by the SUD task (D). From "The Psychological Organization of "Uncertainty" Responses and "Middle" Responses: A Dissociation in Capuchin Monkeys (Cebus apella)," by M. J. Beran, J. D. Smith, M. V. C. Coutinho, J. J. Couchman, and J Boomer, 2009, Journal of Experimental Psychology: Animal Behavior Processes, 35, p. 377. Copyright 2009 by the American Psychological Association. Reprinted with permission. 
In a follow-up study that included capuchin monkeys and macaques, Beran, Perdue, and Smith (2014) arranged a size discrimination task that included a target shape and five foil shapes that differed in size from the target. The correct primary response was to choose the largest shape. The UR would decline that trial and bring a new, randomly selected trial. In this species comparison, macaques still used the UR more frequently than capuchin monkeys on challenging trials (in which the difference between the size of target and foil shapes was small). However, capuchin monkeys did appropriately use the UR more frequently than they had in Beran et al. (2009).

Beran, Perdue, Church, and Smith (2016) tested the hypothesis that the capuchin monkeys' failure at using the UR in Beran et al. (2009) was due to their greater tolerance-compared to macaques-for receiving the punitive timeout for making an incorrect response. Whereas the contingencies granted a 0.50 probability of obtaining a pellet with random selections in Beran et al. (2009), in Beran et al. (2014) the contingency only granted a .166 probability of receiving a pellet for guessing. Perhaps this explained why capuchin monkeys now used the UR more effectively. Accordingly, Beran et al. (2016) had capuchin monkeys engage a shape-size discrimination task with an escape response (similar to Beran et al., 2014), but in blocks of 1,500 trials the number of foils alternated between one foil ( 0.50 probability of a successful guess) and five foils ( 0.166 probability of a successful guess). The capuchin monkey did not use the UR in the 1-foil trials, but did use it more with the 5-foil trials. However, even with the 5-foil trials, these capuchin monkeys only used the UR on approximately $20-25 \%$ of the occasions on the most difficult trials.

Recently, rats have shown some positive evidence for metacognition using a similar manipulation (Yuki \& Okanoya, 2017). In a delayed matching-to-position task, rats were more likely to decline a test (i.e., use a response to ensure that only the correct comparison was presented, but at the cost of a reduced amount of reward) when six comparison stimuli were used versus when two comparison stimuli were used. Thus, failures in finding evidence for metacognition in species other than capuchin monkeys may also be due to the lack of procedures sensitive enough to detect it.

\section{A Meaningful Failure by Capuchin Monkeys, or Failure to Devise a Meaningful Test?}

We are left then with trying to account for why capuchin monkeys seemingly fail where other primates succeed, and whether these failures reflect deficits in metacognition or deficits in methodological approach. We will address first the idea of deficits in metacognition, and then deficits in methodology and assessment.

Perhaps capuchin monkeys lack (or barely register) the experience of difficulty, indecision, and inadequacy. These mental experiences might be inaccessible to whatever working awareness or conscious experiences they may have. This would rule out that they could map adaptive behaviors such as the UR onto situations in which they are at risk of making errors. Or, as seems more likely from the preponderance of the evidence to date, they may have "hints" or "whiffs" of such difficulty, and may occasionally and imperfectly learn how to use response options to mitigate some of their fallibility. But, unlike macaques in some cases, apes in many cases, and humans in nearly all cases, capuchins cannot consistently and efficiently avoid perceptual and cognitive traps by engaging metacognitive monitoring processes and/or control processes. If this is truly a deficit in metacognitive capacity in this species, it begs a broader comparative assessment, especially within the primates, and especially focusing on the New World monkey species. It would be highly significant if it did turn out that reflective mind and mental capacities like metacognition had emerged selectively or more strongly in the Old World lineages. However, we also point out that the success of apes and Old World monkeys itself is a limited-species phenomenon, and so we advocate for data collection with other Old World monkeys as well. Then the data could more clearly indicate a true divide among species that would indicate that metacognitive abilities emerged (or became much more efficient) after the Old World-New World evolutionary split.

Alternatively, perhaps the tasks used with apes, rhesus monkeys, and humans are not sensitive enough to demonstrate capuchin monkey metacognition. Existing data may reflect this possibility. Beran 
et al. $(2014,2016)$ showed that capuchin monkeys may be more risk tolerant than other species given discrimination tasks with a 50\% chance level, and therefore may not engage control mechanisms even in the presence of some form of monitoring process unless the likelihood of failure is too high. Capuchin monkeys are known to be highly manipulative and engage in a variety of foraging behaviors, suggesting perhaps that ecological pressures have selected for a certain degree of risk tolerance in that species. However, we do not know of any systematic assessments of risk tolerance in rhesus macaques and capuchin monkeys that takes into account ecological differences in these species. That may be a productive line of inquiry.

In all species, including humans, one could use a variety of manipulations to increase or decrease the frequency of responses that one considers to be metacognitive. For example, no monkey would escape difficult trials if there were no cost to being wrong. Every human would "phone a friend" to seek information if their life were on the line. So perhaps the data from Beran et al. $(2014,2016)$ show that the degree of risk is a crucial factor for inducing control processes within a metacognitive but risk-tolerant system. The "glimmerings" of metacognitive-like patterns shown by capuchin monkeys by different teams with different tasks that were outlined earlier all appear also to support this possibility, given that in nearly all of those tasks, the costs for failure were not high relative to normal research methods in comparative cognition research. Thus, future research with this species may need to accommodate the degree of risk tolerance shown by its individuals, and this is a point that applies to studies with other species as well. In nearly all studies, there are individual differences that are reported, and an important next step in understanding the evolution of metacognition will come from accounting for those differences across individuals, and across contexts, and across species. We point out that the evolutionary perspective and the methodological perspective taken here are not really in conflict, but could be complementary and mutually confirmatory. That is, if capuchin metacognition is empirically shy and difficult for them to access and use effectively for phylogenetic reasons, then it might require more extreme risk situations to promote the cognitive effort and mental resources that are devoted to its limited use. For this reason, despite their limited successes to date, or perhaps because of those limited successes, capuchin monkeys remain an intriguing species as the field pursues a full evolutionary account of metacognition.

\section{Acknowledgements}

Support for the research from our laboratory discussed in this article was provided by the National Institutes of Health (NICHD grants PO1HD-060563 and RO1HD-061455). We thank our colleagues who have contributed to many of the studies of animal metacognition we have conducted, and especially David Washburn, Barbara Church, Bonnie Perdue, Joseph Boomer, Justin Couchman, and Mariana Coutinho.

\section{References}

Basile, B. M., Hampton, R. R., Suomi, S. J., \& Murray, E. A. (2009). An assessment of memory awareness in tufted capuchin monkeys (Cebus apella). Animal Cognition, 12, 169-180.

Basile, B. M., Schroeder, G. R., Brown, E. K., Templer, V. L., \& Hampton, R. R. (2015). Evaluation of seven hypotheses for metamemory performance in rhesus monkeys. Journal of Experimental Psychology: General, 144, 85-102.

Beran, M. J., Brandl, J., Perner, J., \& Proust, J. (2012). Foundations of metacognition. Oxford, UK: Oxford University Press.

Beran, M. J., Perdue, B. M., Church, B. A., \& Smith, J. D. (2016). Capuchin monkeys (Cebus apella) modulate their use of an uncertainty response depending on risk. Journal of Experimental Psychology: Animal Learning and Cognition, 42, 32-43. 
Beran, M. J., Perdue, B. M., Futch, S. E., Smith, J. D., Evans, T. A., \& Parrish, A. E. (2015). Go when you know: Chimpanzees' confidence movements reflect their responses in a computerized memory task. Cognition, 142, 236-246.

Beran, M. J., Perdue, B. M., \& Smith, J. D. (2014). What are my chances? Closing the gap in uncertainty monitoring between rhesus monkeys (Macaca mulatta) and capuchin monkeys (Cebu apella). Journal of Experimental Psychology: Animal Learning and Cognition, 40, 303-316.

Beran, M. J., \& Smith, J. D. (2011). Information seeking by rhesus monkeys (Macaca mulatta) and capuchin monkeys (Cebus apella). Cognition, 120, 90-105.

Beran, M. J., Smith, J. D., Coutinho, M. V., Couchman, J. J., \& Boomer, J. (2009). The psychological organization of "uncertainty" responses and "middle" responses: A dissociation in capuchin monkeys (Cebus apella). Journal of Experimental Psychology: Animal Behavior Processes, 35, 371-381.

Beran, M. J., Smith, J. D., \& Perdue, B. M. (2013). Language-trained chimpanzees (Pan troglodytes) name what they have seen but look first at what they have not seen. Psychological Science, 24, 660-666.

Brown, E. K., Templer, V. L., \& Hampton, R. R. (2017). An assessment of domain-general metacognitive responding in rhesus monkeys. Behavioural Processes, 135, 132-144.

Call, J. (2010). Do apes know that they could be wrong? Animal Cognition, 13, 689-700.

Call, J., \& Carpenter, M. (2001). Do apes and children know what they have seen? Animal Cognition, 3, $207-220$.

Carruthers, P. (2008). Meta-cognition in animals: A skeptical look. Mind \& Language, 23, 58-89.

Castro, L., \& Wasserman, E. A. (2013). Information-seeking behavior: Exploring metacognitive control in pigeons. Animal Cognition, 16, 241-254.

Dunlosky, J., \& Bjork, R. A. (2008). Handbook of metamemory and memory. New York: Psychology Press.

Flavell, J. H. (1979). Metacognition and cognitive monitoring: A new area of cognitive-developmental inquiry. American Psychologist, 34, 906 -911.

Foote, A. L., \& Crystal, J. D. (2007). Metacognition in the rat. Current Biology, 17, 551-555.

Foote, A. L., \& Crystal, J. D. (2012). "Play it again": A new method for testing metacognition in animals. Animal Cognition, 15, 187-199.

Fujita, K. (2009). Metamemory in tufted capuchin monkeys (Cebus apella). Animal Cognition, 12, 575-585.

Goto, K., \& Watanabe, S. (2012). Large-billed crows (Corvus macrorhynchos) have retrospective but not prospective metamemory. Animal Cognition, 15, 27-35.

Hampton, R. R. (2001). Rhesus monkeys know when they remember. Proceedings of the NationalAcademyof Sciences, 98, 5359-5362.

Hampton, R. R. (2009). Multiple demonstrations of metacognition in nonhumans: Converging evidence or multiple mechanisms? Comparative Cognition \& Behavior Reviews, 4, 17-28.

Hampton, R. R., Zivin, A., \& Murray, E. A. (2004). Rhesus monkeys (Macaca mulatta) discriminate between knowing and not knowing and collect information as needed before acting. Animal Cognition, 7, 239-246.

Inman, A., \& Shettleworth, S. J. (1999). Detecting metamemory in nonverbal subjects: A test with pigeons. Journal of Experimental Psychology: Animal Behavior Processes, 25, 389-395.

Kirk, C. R., McMillan, N., \& Roberts, W. A. (2014). Rats respond for information: Metacognition in a rodent? Journal of Experimental Psychology: Animal Learning and Cognition, 40, 249-259.

Kornell, N., Son, L. K., \& Terrace, H. S. (2007). Transfer of metacognitive skills and hint seeking in monkeys. Psychological Science, 18, 64-71.

Maestripieri, D. (Ed.) (2003). Primate psychology. Cambridge, MA: Harvard University Press.

Marsh, H. L. (2014). Metacognitive-like information seeking in lion-tailed macaques: A generalized search response after all? Animal Cognition, 17, 1313-1328.

Marsh, H. L., \& MacDonald, S. E. (2012a). Information seeking by orangutans: A generalized search strategy? Animal Cognition, 15, 293-304.

Marsh, H. L., \& MacDonald, S. E. (2012b). Orangutans (Pongo abelii) "play the odds": Information-seeking strategies in relation to cost, risk, and benefit. Journal of Comparative Psychology, 126, 263-278.

Metcalfe, J., \& Kober, H. (2005) Self-reflective consciousness and the projectable self. In H. S. Terrace \& J. Metcalfe (Eds.), The missing link in cognition: Origins of self-reflective consciousness (pp. 57-83). New York: Oxford University Press.

Morgan, G., Kornell, N., Kornblum, T., \& Terrace, H. S. (2014). Retrospective and prospective metacognitive judgments in rhesus macaques (Macaca mulatta). Animal Cognition, 17, 249-257.

Nelson, T. O. (1996). Consciousness and metacognition. American Psychologist, 51, 102-116. 
Nelson, T. O., \& Narens, L. (1990). Metamemory: A theoretical framework and new findings. In G. Bower (Ed.), The psychology of learning and motivation: Advances in research and theory (Vol. 26, pp. 125-173). New York: Academic Press.

Paukner, A., Anderson, J. R., \& Fujita, K. (2006). Redundant food searches by capuchin monkeys (Cebus apella): A failure of metacognition? Animal Cognition, 9, 110-117.

Roberts, W. A., Feeney, M. C., McMillan, N., MacPherson, K., Musolino, E., \& Petter, M. (2009). Do pigeons (Columba livia) study for a test? Journal of Experimental Psychology: Animal Behavior Processes, 35, 129-142.

Roberts, W. A., McMillan, N., Musolino, E., \& Cole, M. (2012). Information seeking in animals: Metacognition. Comparative Cognition \& Behavior Reviews, 7, 85-109.

Schwartz, B. L. (2008). Working memory load differentially affects tip-of-the-tongue states and feeling-of-knowing judgments. Memory \& Cognition, 36, 9-19.

Shields, W. E., Smith, J. D., \& Washburn, D. A. (1997). Uncertain responses by humans and rhesus monkeys (Macaca mulatta) in a psychophysical same-different task. Journal of Experimental Psychology: General, $126,147-164$.

Smith, J. D., Beran, M. J., Couchman, J. J., Coutinho, M. V. C., \& Boomer, J. (2009a). Animal metacognition: Problems and prospects. Comparative Cognition and Behavior Reviews, 4, 40-53.

Smith, J. D., Beran, M. J., Couchman, J. J., Coutinho, M. V., \& Boomer, J. B. (2009b). The curious incident of the capuchins. Comparative Cognition and Behavior Reviews, 4, 61-64.

Smith, J. D., Beran, M. J., Redford, J. S., \& Washburn, D. A. (2006). Dissociating uncertainty responses and reinforcement signals in the comparative study of uncertainty monitoring. Journal of Experimental Psychology: General, 135, 282-297.

Smith, J. D., Couchman, J. J., \& Beran, M. J. (2012). The highs and lows of theoretical interpretation in animalmetacognition research. Philosophical Transactions of the Royal Society of London B: Biological Sciences, 367, 1297-1309.

Smith, J. D., Couchman, J. J., \& Beran, M. J. (2014). Animal metacognition: A tale of two comparative psychologies. Journal of Comparative Psychology, 128, 115-131.

Smith, J. D., Redford, J. S., Beran, M. J., \& Washburn, D. A. (2010). Rhesus monkeys (Macaca mulatta) adaptively monitor uncertainty while multi-tasking. Animal Cognition, 13, 93-101.

Smith, J. D., Schull, J., Strote, J., McGee, K., Egnor, R., \& Erb, L. (1995). The uncertain response in the bottlenosed dolphin (Tursiops truncatus). Journal of Experimental Psychology: General, 124, 391-408.

Smith, J. D., Shields, W. E., Schull, J., \& Washburn, D. A. (1997). The uncertain response in humans and animals. Cognition, 62, 75-97.

Smith, J. D., Shields, W. E., \& Washburn, D. A. (2003). The comparative psychology of uncertainty monitoring and metacognition. Behavioral and Brain Sciences, 26, 317-339.

Suda-King, C. (2008). Do orangutans (Pongo pygmaeus) know when they do not remember? Animal Cognition, 11, 21-42.

Suda-King, C., Bania, A. E., Stromberg, E. E., \& Subiaul, F. (2013). Gorillas' use of the escape response in object choice memory tests. Animal Cognition, 16, 65-84.

Teller, S. A. (1989). Metamemory in the pigeon: Prediction of performance on a delayed matching to sample task (Unpublished undergraduate thesis). Reed College, Portland, Oregon.

Templer, V. L., \& Hampton, R. R. (2012). Rhesus monkeys (Macaca mulatta) show robust evidence for memory awareness across multiple generalization tests. Animal Cognition, 15, 409-419.

Templer, V. L., Lee, K. A., \& Preston, A. J. (2017). Rats know when they remember: Transfer of metacognitive responding across odor-based delayed match-to-sample tests. Animal Cognition, 20, 891-906.

Vining, A. Q., \& Marsh, H. L. (2015). Information seeking in capuchins (Cebus apella): A rudimentary form of metacognition? Animal Cognition, 18, 667-681.

Vonk, J., \& Shackelford, T. (Eds.) (2012). Oxford handbook of comparative evolutionary psychology. New York, NY, Oxford University Press.

Watanabe, A., \& Clayton, N. S. (2016). Hint-seeking behaviour of western scrub-jays in a metacognition task. Animal Cognition, 19, 53-64.

Yuki, S., \& Okanoya, K. (2017). Rats show adaptive choice in a metacognitive task with high uncertainty. Journal of Experimental Psychology: Animal Learning and Cognition, 43, 109-118.

Zentall, T. R., \& Stagner, J. P. (2010). Pigeons prefer conditional stimuli over their absence: A comment on Roberts et al.(2009). Journal of Experimental Psychology: Animal Behavior Processes, 36, 506-509. 
Zakrzewski, A. C., Perdue, B. M., Beran, M. J., Church, B. A., \& Smith, J. D. (2014). Cashing out: The decisional flexibility of uncertainty responses in rhesus macaques (Macaca mulatta) and humans (Homo sapiens). Journal of Experimental Psychology: Animal Learning and Cognition, 40, 490-501.

Zentall, T., \& Wasserman, E. (Eds.). (2012). Oxford handbook of comparative cognition. Oxford, UK: Oxford University Press. 